\title{
Factor associated with getting HIV tested for infant whose born from HIV infected mothers, Thailand: 2008
}

Pradabporn Duangajna

From $16^{\text {th }}$ International Symposium on HIV and Emerging Infectious Diseases

Marseille, France. 24-26 March 2010

\section{Background}

Thai National PMTCT program was started since 2001 to provide antiviral drug for pregnant women to cutting HIV transmission from mother in new born children. 2004 PMTCT policy was revised again to launch a new regimen by adding Nevirapine, and High Active Anti Retroviral Therapy for one whose CD4 count less than 200 cell/microlitre. In late 2006 tail end regimen was added to prevent Nevirapine resistant. Antibody and antigen test were done to confirm HIV infection status of children. This study was designed to find out the factor associated with getting HIV tested of children who was born from infected mother.

\section{Methods}

This cross-sectional survey was conducted from March to July 2008. Study population was children whose born from HIV infected mother during October 2006 to December 2007 in 12 provinces with the highest number of HIV-positive delivered from 11 Public Health Regions of Thailand. The total number of sample was 187 to conduct indepth interview.

\section{Results}

Mothers of new born child had ANC performance as 95\%. From that statistics $96 \%$ received counseling about HIV testing of their child, 97\% wanted know their child status, $83 \%$ willing to bring their child for testing, 56\% knew their child result and $94 \%$ were recommended to bring their child for testing at the age of $2,4,6,12$, 18 month. Totally of new born children have HIV Testing is $68 \%$ ( $85 \%$ of by PCR and $15 \%$ by ELISA ) only

Correspondence: pradabpornd@yahoo.com

Ofice of Disease Prevention and Control Region 3 Chonburi Thailand, Chonburi, Thailand
$54 \%$ of them can diagnose their status. The reason of did not receive testing because of did not met criteria for testing(47\%), miss an appointment, no information available, sicked or died before testing( $2 \%)$, lack of money for commutation, did not want to get testing, could not draw blood, loss to follow up(22\%), children did not live with their parents. The important things were their parents did not getting to continuous care services and did not want to disclosure their status.

\section{Discussion}

Although mothers reported high pre-post test counseling uptake(96\%), high HIV \& PMTCT knowledge scores (8.6/10 points), high uptake of ARV for PMTCT(94\%) and access to HIV care services. But some of them need more HIV information and confidentiality protection concerns. In case of lower coverage of early infant HIV testing the potential setting should be improved especially surveillance system for these children and Laboratory unit should be covered overall health care unit to support for PCR or ELISA testing. Not only that Information, Education and Communication are important as well to bring the children come to get testing and prevent of miss or delay testing. Finally to increase coverage of HIV testing in new born their mothers should have a plan to follow up and mobile testing should be prepared for this situation.

Published: 11 May 2010

doi:10.1186/1742-4690-7-S1-P125

Cite this article as: Duangajna: Factor associated with getting HIV tested for infant whose born from HIV infected mothers, Thailand: 2008. Retrovirology 2010 7(Suppl 1):P125. 